OPEN ACCESS

Edited by:

Urszula Krzych,

Walter Reed Army Institute of

Research, United States

Reviewed by:

Giampietro Corradin,

University of Lausanne, Switzerland

Joshua Tan,

National Institutes of Health $(\mathrm{N} / \mathrm{H})$,

United States

*Correspondence:

Jo-Anne Chan

jo-anne.chan@burnet.edu.au

James G. Beeson

beeson@burnet.edu.au

${ }^{t}$ These authors have contributed equally to this work and share first authorship

${ }^{\ddagger}$ These authors have contributed equally to this work and share

senior authorship

Specialty section:

This article was submitted to

Vaccines and

Molecular Therapeutics,

a section of the journal

Frontiers in Immunology

Received: 14 December 2020

Accepted: 01 March 2021

Published: 17 March 2021

Citation:

Kurtovic L, Wetzel D, Reiling L, Drew DR, Palmer C, Kouskousis $B$ Hanssen $E$, Wines $B D$, Hogarth PM, Suckow $M$, Jenzelewski $V$, Piontek $M$, Chan J-A and Beeson JG (2021) Novel Virus-Like Particle Vaccine Encoding the Circumsporozoite Protein of

Plasmodium falciparum is Immunogenic and Induces Functional Antibody Responses in Mice.

Front. Immunol. 12:641421. doi: 10.3389/fimmu.2021.641421

\section{Novel Virus-Like Particle Vaccine Encoding the Circumsporozoite Protein of Plasmodium falciparum Is Immunogenic and Induces Functional Antibody Responses in Mice}

\author{
Liriye Kurtovic ${ }^{1,2 \dagger}{ }^{\text {, David Wetzel }}{ }^{3 \dagger}$, Linda Reiling ${ }^{1}$, Damien R. Drew ${ }^{1}$, Catherine Palmer ${ }^{1}$, \\ Betty Kouskousis ${ }^{1}$, Eric Hanssen ${ }^{4}$, Bruce D. Wines ${ }^{1,2,5}$, P. Mark Hogarth ${ }^{1,2,5}$, \\ Manfred Suckow ${ }^{3}$, Volker Jenzelewski ${ }^{3}$, Michael Piontek ${ }^{3}$, Jo-Anne Chan ${ }^{1,2,6 * \neq}$ \\ and James G. Beeson ${ }^{1,2,6,7 * \neq}$ \\ ${ }^{1}$ Life Sciences, Burnet Institute, Melbourne, VIC, Australia, ${ }^{2}$ Departments of Immunology and Pathology and Infectious \\ Diseases, Central Clinical School, Monash University, Melbourne, VIC, Australia, ${ }^{3}$ ARTES Biotechnology GmbH, Langenfeld, \\ Germany, ${ }^{4}$ The Bio21 Molecular Science and Biotechnology Institute, The University of Melbourne, Parkville, VIC, Australia, \\ ${ }^{5}$ Clinical Pathology, The University of Melbourne, Parkville, VIC, Australia, ${ }^{6}$ Department of Medicine, Royal Melbourne \\ Hospital, The University of Melbourne, Parkville, VIC, Australia, ${ }^{7}$ Department of Microbiology, Monash University, Clayton, \\ VIC, Australia
}

RTS,S is the leading malaria vaccine in development, but has demonstrated only moderate protective efficacy in clinical trials. RTS,S is a virus-like particle (VLP) that uses the human hepatitis B virus as scaffold to display the malaria sporozoite antigen, circumsporozoite protein (CSP). Particle formation requires four-fold excess scaffold antigen, and as a result, CSP represents only a small portion of the final vaccine construct. Alternative VLP or nanoparticle platforms that reduce the amount of scaffold antigen and increase the amount of the target CSP antigen present in particles may enhance vaccine immunogenicity and efficacy. Here, we describe the production and characterization of a novel VLP that uses the small surface antigen (dS) of duck hepatitis B virus to display CSP. The CSP-dS fusion protein successfully formed VLPS without the need for excess scaffold antigen, and thus CSP represented a larger portion of the vaccine construct. CSP-dS formed large particles approximately 31-74 nm in size and were confirmed to display CSP on the surface. CSP-dS VLPS were highly immunogenic in mice and induced antibodies to multiple regions of CSP, even when administered at a lower vaccine dosage. Vaccine-induced antibodies demonstrated relevant functional activities, including Fc-dependent interactions with complement and Fcy-receptors, previously identified as important in malaria immunity. Further, vaccine-induced antibodies had similar properties (epitope-specificity and avidity) to monoclonal antibodies that are protective in mouse models. Our novel platform to produce VLPs without excess scaffold protein has wide implications for the future development of vaccines for malaria and other infectious diseases.

Keywords: circumsporozoite protein (CSP), duck hepatitis B surface antigen, malaria, Plasmodium falciparum, RTS,S, vaccines, virus-like particle (VLP) 


\section{INTRODUCTION}

There were an estimated 228 million cases of malaria and 405,000 deaths in 2018, largely attributed to infection with Plasmodium falciparum (1). Antimalarial interventions currently available include vector control measures to prevent transmission of the malaria-causing parasite from mosquitoes to humans, and drugs to clear infection and treat clinical illness. However, the effectiveness of these tools is threatened by the emergence and spread of drug and insecticide resistance (2), and disruptions to and gaps in public health interventions (3). A critical step towards reducing malaria burden and achieving global malaria elimination will require innovative research to develop new interventions, including efficacious malaria vaccines (4).

The most advanced malaria vaccine in development is RTS,S, which is based on the major surface antigen expressed by $P$. falciparum sporozoites, the circumsporozoite protein (CSP). This vaccine approach aims to prevent the initial asymptomatic stage of infection in the liver and to therefore prevent subsequent parasite replication in the blood and development of clinical disease (5). The RTS,S vaccine construct is a fusion protein of a truncated form of CSP (including the central-repeat and C-terminal regions of the protein) and the human hepatitis B surface antigen (HBsAg), which is co-expressed with excess HBsAg to self-assemble into virus-like particles (VLPs) (6). These particles are $\sim 20 \mathrm{~nm}$ in size and co-administered with the potent AS01 adjuvant to enhance vaccine immunogenicity (7).

RTS,S/AS01E is the only malaria vaccine to complete phase III clinical trials, which were conducted at 11 study sites in subSaharan Africa. RTS,S vaccination conferred 25.9\% (95\% confidence interval, $19.9 \%$ to $31.5 \%$ ) and $36.3 \%$ (confidence interval, $31.8 \%$ to $40.5 \%$ ) protective efficacy against clinical malaria in infants and children, respectively, after 3-4 years (8). However, vaccine efficacy was initially high in the first year (around 50\%), but waned substantially in the 18 months after completion of primary vaccination of three doses (9). To further evaluate vaccine safety and efficacy, pilot implementation of RTS, $S$ recently commenced in Ghana, Kenya and Malawi as recommended by the World Health Organization (WHO) (10). RTS,S vaccine-induced antibodies are thought to primarily mediate protective immunity (5), and the magnitude of antiCSP IgG broadly correlates with vaccine efficacy (11). While the precise mechanisms involved are unclear, some antibody properties and functional activities have been associated with clinical protection, including IgG avidity, complement-fixation,

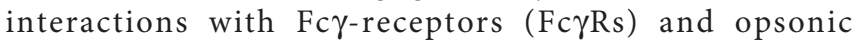
phagocytosis by neutrophils (12-15). Inhibiting sporozoite invasion of hepatocytes is another proposed antibody mechanism from in vitro studies (5), although, this has not been established as a correlate of protection for RTS,S or other CSP-based vaccines in humans. While RTS,S is the leading malaria vaccine candidate, it is an imperfect vaccine that is unlikely to drive malaria elimination as a stand-alone tool. Strategies to modify RTS,S could lead to the development of RTS,S-like or next-generation vaccines with improved immunogenicity, efficacy and longevity in target populations. An important limitation of the RTS,S vaccine construct is the need for excess HBsAg for particle formation to occur and thus, the resulting VLP is comprised of only $20 \%$ CSP-HBsAg fusion protein and $\sim 80 \%$ unfused HBsAg scaffold protein (16). A novel CSP-based particle vaccine was recently described known as R21, which did not require excess unfused HBsAg for particle formation, and therefore had an increased proportion of CSP compared to RTS,S. R21 was immunogenic and efficacious in a pre-clinical murine vaccine study, and may improve upon current vaccine approaches (17). Another consideration is the VLP scaffold protein used to display the CSP antigen, which can influence particle size and structure, and therefore influence the induction of protective immune responses (18).

Here we describe the production and characterization of a novel VLP-based vaccine candidate that displays CSP on the surface. We used the membrane integral small surface protein (dS) of the duck hepatitis B virus (DHBV) as a VLP scaffold protein (19-21). As a proof-of-concept for this vaccine platform, the same truncated form of CSP that is included in RTS,S (central-repeat and C-terminal regions of the protein) was selected as the malaria vaccine antigen and was genetically fused to the $\mathrm{dS}$. The resulting CSP-dS fusion protein was overexpressed in recombinant yeast Hansenula polymorpha, which is a cell line suitable for large-scale vaccine manufacturing. This approach resulted in the formation of VLPs with the CSP-dS fusion protein alone, without the need for excess $\mathrm{dS}$ as a scaffold protein, thereby achieving a higher ratio of the CSP vaccine antigen compared to the approach used for RTS,S and other VLP-based vaccines. The biophysical properties of these VLPs were characterized and the expression of CSP confirmed through Western blotting, transmission electron microscopy and super resolution microscopy. We performed an immunogenicity study of the CSP-dS VLPs in mice and measured the ability of vaccine-induced antibodies to interact with complement and Fc $\gamma$ Rs, which have been associated with RTS,S vaccine efficacy (12), as well as IgG avidity, and the ability of antibodies to compete with a human monoclonal antibody $(\mathrm{mAb})$ that confers protection in a mouse model.

\section{MATERIALS AND METHODS}

\section{Generation and Purification of the CSP-dS VLP}

The CSP-dS fusion protein was composed of a truncated form of $P$. falciparum CSP including amino acids 199-387 (XP_001351122.1) (22) N-terminally fused to the scaffold protein dS (Genbank accession number: MF510122, Figure 1). The resulting CSP-dS encoding gene (Genbank accession number: MH142263) was synthesized by GeneArt/Life Technologies (Regensburg, Germany) and codon-optimized for heterologous expression in $H$. polymorpha. The sequence was subcloned into a derivative of the $H$. polymorpha expression plasmid pFPMT121 (23) which carried the LEU2 instead URA3 gene for selection in yeast. The auxotrophic $H$. polymorpha strain 


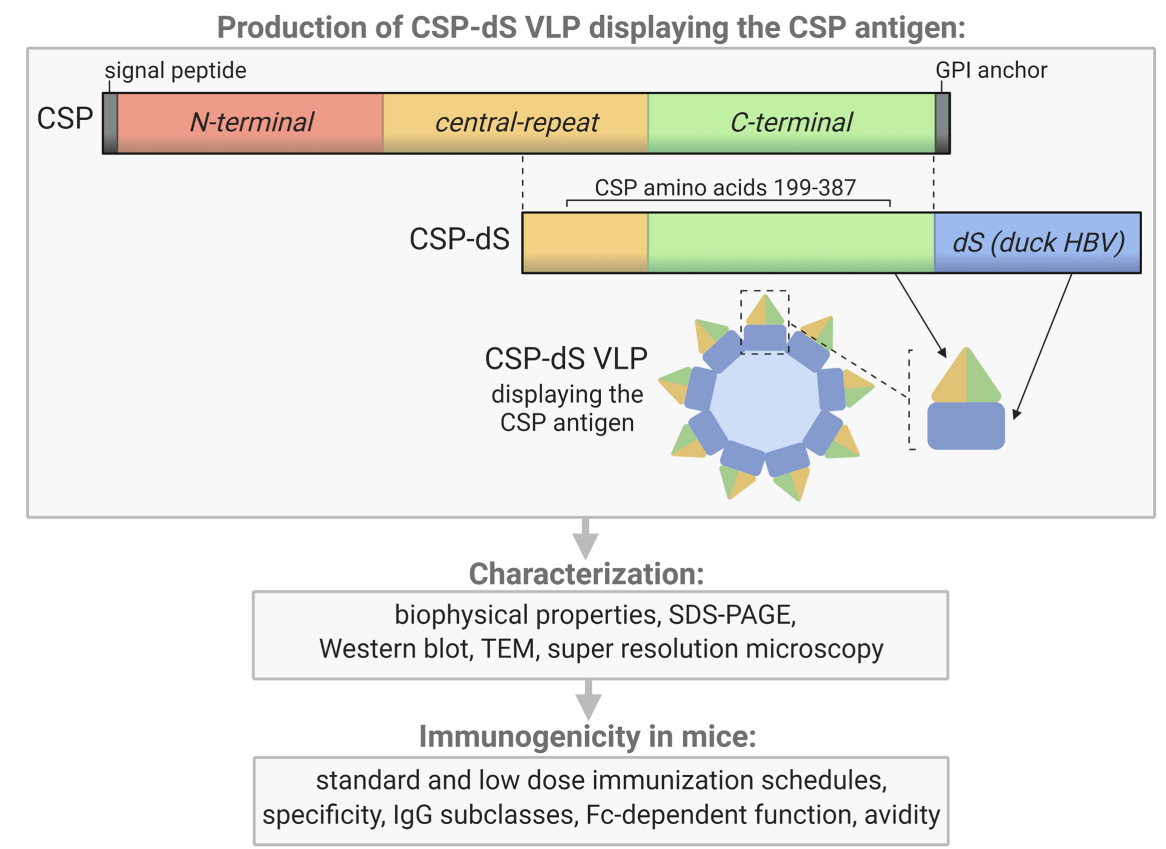

FIGURE 1 | Schematic of the CSP-dS VLP and summary of the study workflow. CSP-dS VLPS were produced by genetically fusing a truncated form of CSP (including the central-repeat and C-terminal regions, amino acids 199-387) to the small surface protein (dS) of the duck hepatitis B virus (HBV). The CSP-dS fusion protein formed virus-like particles, which were characterized and tested for immunogenicity in mice. Created with BioRender.com.

ALU3 (relevant genotype: ade1, leu2, ura3) (24) derived from wild type strain ATCC ${ }^{\circledR} 34438^{\text {TM }}$ (CBS 4732, IFO 1476, JCM 3621, NBRC 1476, NCYC 1457, NRRL Y-5445) was used as an expression host. Recombinant yeast cell lines were generated by electroporation (25) and a subsequent strain generation and isolation protocol (26). Thereby, the expression plasmid stably integrated into the yeast genome. Heterologous yeast strains were stored as glycerol stocks at $-80^{\circ} \mathrm{C}$.

The production cell line was cultured in $2 \mathrm{~L}$ baffled shake flasks filled with $200 \mathrm{~mL}$ animal component-free YPG medium containing $20 \mathrm{~g} \mathrm{~L}^{-1}$ glycerol (AppliChem, Germany) as a carbon source and $0.1 \mathrm{~g} \mathrm{~L}^{-1}$ adenine (AppliChem). Pre-cultures grown in YPD medium to stationary phase were used as inoculum. Cultures were incubated at $37{ }^{\circ} \mathrm{C}$ and $130 \mathrm{rpm}$ with $5 \mathrm{~cm}$ throw. After $56 \mathrm{~h}$ of batch growth and derepression of the promoter system by consumption of glycerol, $1 \%(\mathrm{v} / \mathrm{v})$ methanol was added to the cultures for induction of target gene expression. After $72 \mathrm{~h}$ total cultivation time, cells were harvested by centrifugation $\left(6,000 \mathrm{~g}, 15 \mathrm{~min}, 4^{\circ} \mathrm{C}\right)$, washed once with wash buffer (50 $\mathrm{mM}$ Na-phosphate buffer, $2 \mathrm{mM}$ EDTA, $\mathrm{pH}$ 8.0) and stored at $-20^{\circ} \mathrm{C}$.

CSP-dS VLPs were isolated by ultracentrifugation as described previously (20). Briefly, cells were disrupted by highpressure homogenization. The soluble material was then layered on top of sucrose cushions ( $2 \mathrm{~mL} 70 \%(\mathrm{w} / \mathrm{v}) ; 3 \mathrm{~mL} \mathrm{20 \% (w/v))}$ and the boundary layers between the two sucrose layers were harvested after ultracentrifugation $\left(90 \mathrm{~min}, 51,000 \mathrm{rpm}, 18^{\circ} \mathrm{C}\right.$,
Optima $^{\text {TM }}$ L90K centrifuge, rotor type: $70.1 \mathrm{Ti}$, tubes: $16 * 76 \mathrm{~mm}$, Beckman Coulter, USA). These fractions were subsequently mixed with $6 \mathrm{M} \mathrm{CsCl}$ (AppliChem) stock solution to $1.5 \mathrm{M}$ final $\mathrm{CsCl}$ concentration. Mixtures were subjected to density gradient separation $\left(65 \mathrm{~h}\right.$ at $48,400 \mathrm{rpm}, 4^{\circ} \mathrm{C}$ ). Product containing fractions were pooled and desalted by dialysis (SlideA-Lyzer ${ }^{\mathrm{TM}}$ dialysis cassettes, MWCO $20 \mathrm{kDa}$, Thermo Fisher Scientific, USA) against desalting buffer (8 $\mathrm{mM} \mathrm{Na-phosphate}$

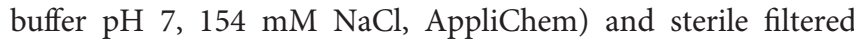
(Filtropur S 0.2 filters, Sarstedt, Germany).

\section{Visualization of CSP-dS VLPs}

CSP-dS VLPs were visualized by negative staining transmission electron microscopy (TEM) and super-resolution microscopy (Structured-Illumination Microscopy, SIM) as previously described $(19,20)$. N-SIM was used to evaluate the co-localization of CSP and the scaffold protein dS in nano-scaled structures, as previously described (20). Samples were dual labelled with $4 \mu \mathrm{g} / \mathrm{mL}$ of primary antibodies (rabbit anti-CSP and mouse anti-dS, 7C12), followed by secondary antibodies (1/1000, anti-rabbit AlexaFluor 594 and anti-mouse AlexaFluor 488; Thermo Fisher Scientific). The super-resolution images were collected using a Nikon N-SIM microscope equipped with 488, 561 and $640 \mathrm{~nm}$ lasers, an Andor iXON DU897 EM-CCD camera and a 100x oil immersion lens having a numerical aperture of 1.49 . The $\mathrm{z}$-series was acquired using NIS-Elements and analyzed both using NIS-Elements and the open java source, ImageJ/FIJI. 


\section{Western Blots}

CSP-dS VLPs and full-length recombinant CSP (rCSP) were prepared under reducing conditions, separated by SDS-PAGE using $4-12 \%$ Bis-Tris gels (NuPAGE, Thermo Fisher Scientific) and transferred onto nitrocellulose membranes using the iBlot system (Thermo Fisher Scientific) according to the manufacturer instructions. Membranes were blocked with $10 \%$ (w/v) skim milk in phosphate buffered saline (PBS) and probed with polyclonal rabbit anti-CSP IgG $(1 \mu \mathrm{g} / \mathrm{mL})$ and a dS-specific mouse monoclonal antibody $(7 \mathrm{C} 12 ; 1 / 1000)$. This was followed by species-specific detection HRP-conjugated antibodies (1/5000; Millipore, USA). Protein bands were detected using SuperSignal Chemiluminescent HRP substrate (Thermo Fisher Scientific) and imaged using the ChemiDoc System (Bio-Rad, USA).

\section{Evaluation of Particle Size}

Particle size distribution of the CSP-dS VLP preparation was analyzed by dynamic light scattering (DLS) as previously described (20). This analysis was performed before and after analytical high-performance size exclusion chromatography (HP-SEC) using Shimadzu CBM-20A system (Canby, OR, USA) equipped with communication bus modelus (CBM-20A), UV/VIS detector (SPD20-A), degasser (DGU-20A5), liquid chromotograph (LC-20AT), auto sampler (SIL-20AHT) and column oven (CTO-20AC). A volume of $50 \mu \mathrm{L}$ of the CSP-dS VLP preparation was applied to $\mathrm{TSKgel}^{\circledR} \mathrm{G}^{\circledR} 000 \mathrm{PW}_{\mathrm{XL}} 7.8^{\star} 300$ $\mathrm{mm}$ column (Tosoh Bioscience $\mathrm{GmbH}$, Griesheim, Germany) in loading buffer (1.7 mM KH $2 \mathrm{PO}_{4}, 7.9 \mathrm{mM} \mathrm{Na} 2 \mathrm{HPO}_{4}, 2.7 \mathrm{mM}$ $\mathrm{KCl} ; 144 \mathrm{mM} \mathrm{NaCl}, \mathrm{pH} 7.3$ ) at $0.5 \mathrm{ml} / \mathrm{min}$ flow. Absorption of the eluate was tracked with the detector SPD20-A at $214 \mathrm{~nm}$ and $280 \mathrm{~nm}$ wavelength. Note that only a small amount of sample was separated from the mainstream of sample preparation, for the purpose of analysis.

\section{Animal Immunizations}

Mice were immunized with the CSP-dS VLP or full-length rCSP as a non-VLP control (rCSP vaccine) (27). The VLP preparation used was derived from $\mathrm{CsCl}$ density gradient centrifugation followed by dialysis and filtration, which was adapted from a HBsAg VLP production process (28). For the first study, Swiss mice received three $10 \mu \mathrm{g}$ doses of CSP-dS $(n=5)$ or $\operatorname{rCSP}(n=5)$ vaccines with each dose administered 2 weeks apart (via intraperitoneal injection). The terminal bleed was performed 2 weeks after the final dose and the serum was used for immunogenicity studies. The second study was similar except that C57/BL6 mice were used (due to availability) and instead received three $2 \mu \mathrm{g}$ doses of CSP-dS $(n=5)$. For all immunizations, the CSP-dS and $\mathrm{rCSP}$ vaccines were formulated with equal volumes of sterile aluminum hydroxide (Alhydrogel adjuvant; Brenntag, Denmark) and incubated on the shaker for 5 min prior to immunizations. Animal immunizations were conducted at the Animal Facility at the Walter and Eliza Hall Institute (Melbourne, Australia) and ethics approval was obtained by the Animal Ethics Committee of the Walter and Eliza Hall Institute.

\section{Antigens}

The following antigens used in this study were all based on the $P$. falciparum 3D7 sequence: full-length rCSP (excluding the signal peptide and glycosylphosphatidylinositol sequences) expressed in Escherichia coli (Gennova, India) (27), synthetic peptide representative of the central-repeat region of CSP (NANP, Life Tein, USA) (29), and recombinant C-terminal region of CSP expressed in HEK293 cells (CT) (29).

\section{Immunogenicity Assays}

Antibody responses were measured by standard enzyme-linked immunosorbent assay (ELISA) as follows (29). Ninety-six well flat bottom MaxiSorp Nunc plates (Thermo Fisher Scientific) were coated with $0.5 \mu \mathrm{g} / \mathrm{mL}$ antigen (full-length rCSP, NANP and CT) in PBS overnight at $4^{\circ} \mathrm{C}$. Plates were blocked with $1 \%$ $(\mathrm{w} / \mathrm{v})$ casein in $\mathrm{PBS}$ for $2 \mathrm{~h}$ at $37^{\circ} \mathrm{C}$ and then incubated with test mouse serum diluted in buffer $(0.1 \%$ casein in PBS) for $2 \mathrm{~h}$ at room temperature (RT). To measure total IgG, plates were incubated with goat anti-mouse IgG HRP (Millipore) at 1/2000 in buffer for $1 \mathrm{~h}$ at room temperature (RT). Finally, plates were incubated with 2,2'-azino-bis(3-ethylbenzothiazoline-6sulphonic acid) substrate (ABTS, Thermo Fisher Scientific) for 15 min at RT shielded from light and absorbance was measured at optical density (OD) $405 \mathrm{~nm}$ using the Multiskan Go plate reader (Thermo Fisher Scientific). To measure murine IgG subclasses, plates were incubated with goat anti-mouse IgG1, IgG2a, IgG2b or IgG3 detection antibodies (SouthernBiotech, USA) at $1 / 1000$ in buffer, followed by rabbit anti-goat IgG HRP (Sigma-Aldrich, USA) at 1/1000 in buffer, each incubated for $1 \mathrm{~h}$ at RT. Finally, plates were incubated with 3,3',5,5'Tetramethylbenzidine substrate (TMB, Thermo Fisher Scientific) for $5 \mathrm{~min}$ at RT shielded from light; reactivity was stopped using $1 \mathrm{M}$ sulfuric acid and absorbance was measured at OD $450 \mathrm{~nm}$. In all plate-based immunoassays between each incubation step, plates were washed thrice in PBS-Tween20 $0.05 \%(\mathrm{v} / \mathrm{v})$ using the ELx405 automated plate washer (BioTek, USA).

The ability of vaccine-induced antibodies to fix human complement protein, C1q, was performed as previously described $(30,31)$. Briefly, ninety-six well plates were coated, blocked, and incubated with test serum (at 1/100 dilution) as described for standard ELISA. Following on from this, plates were incubated with $10 \mu \mathrm{g} / \mathrm{mL}$ human C1q (Millipore) in buffer for $30 \mathrm{~min}$ at RT. To measure C1q-fixation, plates were then incubated with rabbit anti-C1q IgG (in-house) (29), followed by goat anti-rabbit IgG HRP (Millipore), each at $1 / 2000$ in buffer and incubated for $1 \mathrm{~h}$ at RT. Finally, plates were incubated with TMB substrate for $1 \mathrm{~h}$ at RT shielded from light; reactivity was stopped using $1 \mathrm{M}$ sulfuric acid and absorbance was measured at OD $450 \mathrm{~nm}$.

Vaccine-induced antibodies were also tested for the ability to interact with human FcyRs, as previously described $(12,32)$. Briefly, ninety-six well plates were coated, blocked, and incubated with test serum (at 1/100 dilution) as described for standard ELISA. Plates were incubated with $0.2 \mu \mathrm{g} / \mathrm{mL}$ biotin- 
labelled dimeric recombinant soluble FcyRIIa (H131 allele) (33), and Fc $\gamma \mathrm{R}$-binding was detected using streptavidin conjugated HRP (Thermo Fisher Scientific) at 1/10000 dilution for $1 \mathrm{~h}$. Note that for Fc $\gamma \mathrm{R}$-biding assays, blocking and dilutions were performed using $1 \%(\mathrm{w} / \mathrm{v})$ bovine serum albumin in PBS, and all incubations were conducted at $37^{\circ} \mathrm{C}$. Finally, plates were incubated with TMB substrate for $1 \mathrm{~h}$ at RT shielded from light; reactivity was stopped using $1 \mathrm{M}$ sulfuric acid, and absorbance was measured at OD $450 \mathrm{~nm}$.

\section{Monoclonal Antibody (mAb) Experiments}

Mouse anti-NANP mAb, 2A10 (34), was tested for anti-NANP IgG between 1 and $0.008 \mu \mathrm{g} / \mathrm{ml}$ to generate an 8 -point standard curve using the ELISA method described above. The mAb concentration was $\log (10)$ transformed to determine a linear line of best fit. The resulting equation $(y=0.6921 x+1.447)$ was used to estimate the concentration of NANP-IgG in serum from CSP-dS and rCSP vaccine groups collected after the third $10 \mu \mathrm{g}$ dose. Samples were tested on the same plate at 1/2000, 1/4000 and 1/8000 dilutions, and OD values from all dilutions were used to estimate the IgG concentration. 2A10 was expressed in HEK293T cells and purified by affinity chromatography as previously described (32).

The $2 \mathrm{~A} 10 \mathrm{mAb}(0.5 \mu \mathrm{g} / \mathrm{ml})$ and pooled serum from CSP-dS and rCSP vaccine groups (1/2000 dilution) were tested for IgG avidity to NANP (35). This used a similar method to the ELISA described above, but following sample incubation, ammonium thiocyanate was added to between 0 and $10 \mathrm{M}$, for $20 \mathrm{~min}$ at RT. The OD value in the absence of ammonium thiocyanate (0M) was considered the maximal response, and IgG avidity index was expressed as a percentage of this maximal response, E.g. (10M/ $0 \mathrm{M})^{\star} 100$.

The $2 \mathrm{~A} 10 \mathrm{mAb}(1 \mu \mathrm{g} / \mathrm{ml})$ and serum samples from mice in the CSP-dS vaccine group (1/25 dilution) were tested for the ability to compete with human anti-NANP mAb, MGG4, for binding to NANP (36). The protocol was similar to the ELISA described above and adapted from prior approaches (37), whereby samples were mixed with MGG4 $(0.125 \mu \mathrm{g} / \mathrm{ml})$ prior to plate application. A well containing only MGG4 without competing mouse antibody was also included as a control. Goat anti-human IgG HRP (Invitrogen, 1/1000) was used to detect the human MGG4 mAb. Percent inhibition of MGG4 binding was calculated as (1-(competing antibody/MGG4 alone) )*100. MGG4 (human IgG1) was expressed in HEK293T cells and purified by affinity chromatography as previously described (32).

\section{Statistical Analysis}

Mouse serum samples were tested in duplicate, and raw data were corrected for background reactivity using no-serum wells that were included as a negative control. Results were shown from two independent experiments, unless specified otherwise. For IgG responses measured at multiple serum dilutions, the area under the curve (AUC) was calculated for each mouse and compared between vaccine groups using the unpaired t-test. For IgG subclass and Fc-dependent antibody responses measured at a single dilution, OD values were compared between vaccine groups using the unpaired t-test. We also performed a Pearson's correlation ( $r$ ) correlation analysis on all antibody variables. Data were analyzed using GraphPad Prism 8.

\section{RESULTS}

\section{Production of the CSP-dS VLP}

The recombinant yeast strain, Der\#949 (relevant genotype: ade1, LEU2, ura3) was isolated from transformation of host strain ALU3 with the CSP-dS encoding plasmid. This strain was used to produce the CSP-dS VLPs. The CSP-dS construct contained amino acids 199-387 of $P$. falciparum CSP (including the centralrepeat and C-terminal regions; Figure 1) with the N-terminal MMAP motif and a GPVTN linker between the CSP fragment and the C-terminal dS. Approximately $6 \mathrm{~g}$ dry cell weight (DCW) of yeast strain Der\#949 were used to isolate $1.2 \pm 0.1 \mathrm{mg}$ product VLP containing the CSP-dS fusion protein $\left(\mathrm{Y}_{\mathrm{P} / \mathrm{X}}=\sim 0.2 \mathrm{mg} \mathrm{g}^{-1}\right)$ applying an analytical methodology based on two consecutive steps of ultracentrifugation. The preparation resulted in a clean protein band of approximately $50 \mathrm{kDa}$ in size by SDS-PAGE, indicating there was minimal incorporation of yeast proteins in the VLP preparation as previously shown (20) (Figure 2A). Additionally, the CSP-dS fusion protein was specifically recognized by anti-dS and anti-CSP antibodies by Western blot (Figure 2B).

\section{CSP-dS Formed Particulate Nanostructures Recognized by CSP- Specific Antibodies}

The size distribution analyzed by dynamic light scattering (DLS) revealed a monomodal but polydisperse population of particles (Figure 3A; polydispersity index (PDI): 0.59). The VLP preparation was subsequently analyzed by HP-SEC (Figure S1A) and the product-containing fraction was again analyzed

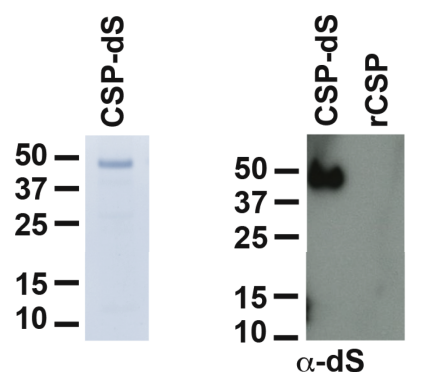

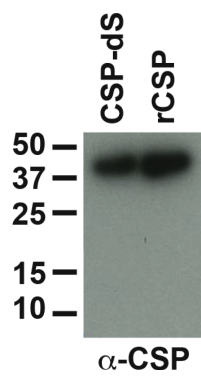

FIGURE 2 | Analyses of purified CSP-dS VLP derived from strain Der\#949. (A) Reducing SDS-PAGE of CSP-dS VLP purified from strain Der\#949 stained with Colloidal blue. A band of expected molecular weight corresponding to CSP-dS was observed at approximately $50 \mathrm{kDa}$. (B) CSPdS VLP and rCSP as a control were prepared for Western blot under reducing conditions. Membranes were probed with a monoclonal anti-dS antibody (7C12) and polyclonal anti-CSP antibody. 

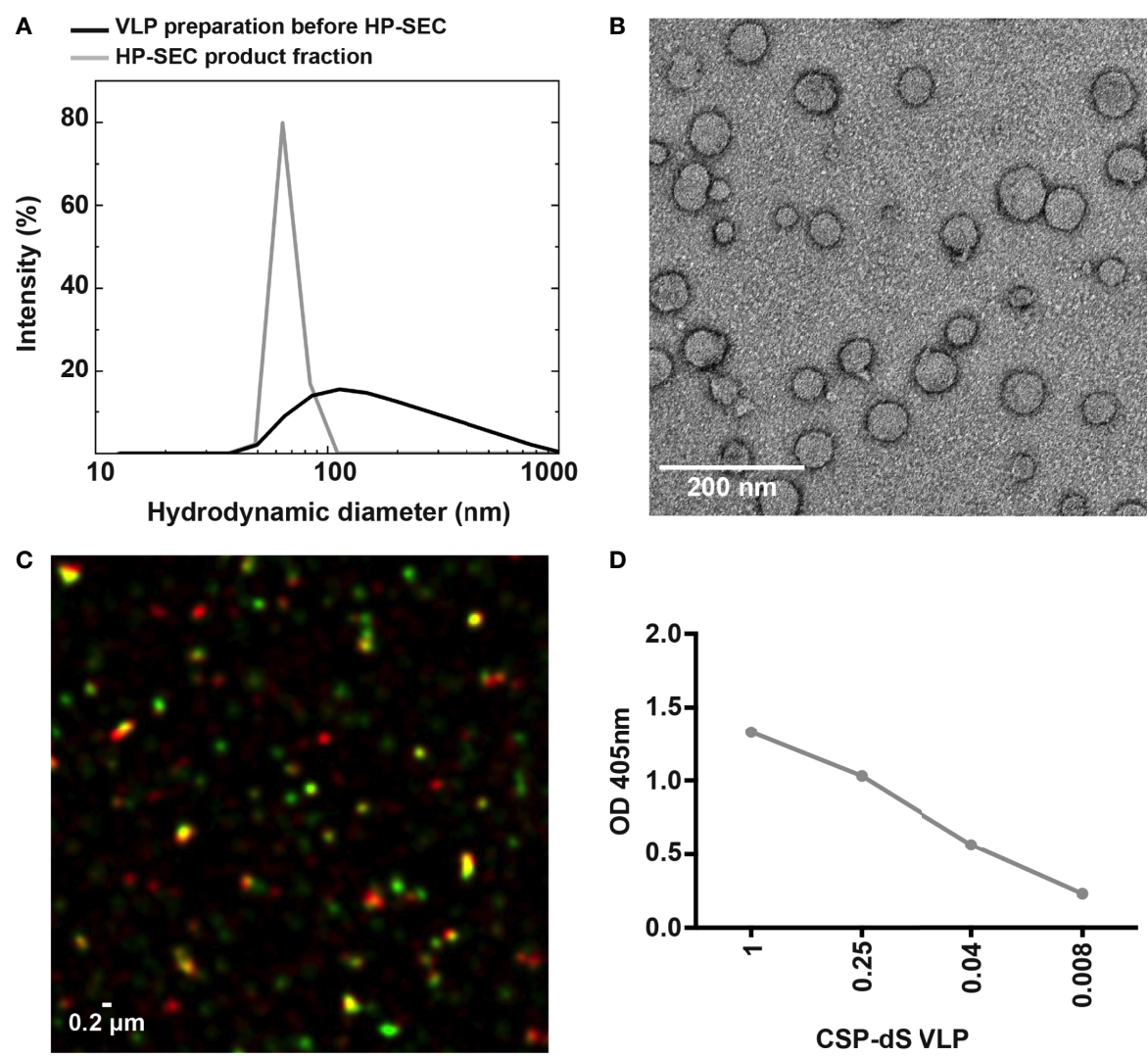

D

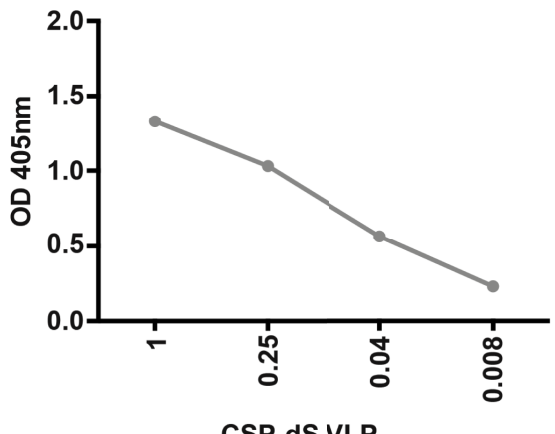

CSP-dS VLP

coating concentration $\mu \mathrm{g} / \mathrm{ml}$

FIGURE 3 | Characterizing the expression of CSP on the VLP surface. (A) Analysis of the particulate character of CSP-dS VLP purified from strain Der\#949. Size distribution was determined by DLS before and after analysis by HP-SEC. (B) The structure of CSP-dS VLPs visualized by negative staining TEM. The VLP structures are consistent with the expected size of approximately $31-74 \mathrm{~nm}$. (C) Super-resolution microscopy (N-SIM) was used to visualize CSP-dS VLPS probed with CSPspecific (red) and dS-specific (green) antibodies. Co-localization of CSP and dS are presented in yellow fluorescence. A representative image is shown, and scale bar represents $0.2 \mu \mathrm{m}$. (D) Binding of polyclonal anti-CSP antibodies to CSP-dS VLP measured by standard ELISA; mean and range of duplicates are shown.

by DLS. The analysis indicated a homogeneous particle population characterized by $73 \mathrm{~nm}$ hydrodynamic diameter (PDI: 0.04). We also confirmed the CSP-dS was highly stable after 6 months of storage at $4-8^{\circ} \mathrm{C}$ by HP-SEC (Figure S1B). Transmission electron microscopy (TEM) was used to characterize the VLPs formed by the CSP-dS fusion protein (Figure 3B). The TEM image showed the formation of homogeneous particles of 31-74 $\mathrm{nm}$ in diameter based on manual evaluation (Figures 3B, S2). The hydrodynamic diameters determined by DLS were slightly larger than the respective diameters specified by manual evaluation of the TEM images, which has been observed previously with other VLP preparations (19). Nevertheless, all data collected were within the dimensions that could be expected for this type of $\operatorname{VLP}(20,38)$. The determined buoyant density $\left(1.14-1.15 \mathrm{~g} \mathrm{~cm}^{-3}\right)$ was also plausible for lipoproteins or VLPs (39). Summary of the production process for CSP-dS can be found in Table $\mathbf{1}$.

The structure of the CSP-dS VLPs were further visualized using super-resolution microscopy (N-SIM; Figure 3C). Polyclonal anti-CSP antibodies were used to detect CSP expression and monoclonal anti-dS antibodies were used to
TABLE 1 | Summary of production process leading CSP-dS VLP preparation.

\begin{tabular}{lc}
\hline Designation of VLP & CSP-dS VLP \\
\hline H. polymorpha strain & Der\#949 \\
Cell mass generation & Shake flask \\
DCW used for VLP purification $[\mathrm{g}]$ & $\sim 6^{(a)}$ \\
Isolated VLP [mg] & $1.2 \pm 0.1$ \\
VLP yield per biomass (DCW), YPX $\left[\mathrm{mg} \mathrm{g}^{-1}\right]$ & $\sim 0.2^{(a)}$ \\
Product yield per culture volume $\left[\mathrm{mg} \mathrm{L}^{-1}\right]$ & $\sim 2^{(a)}$ \\
VLP diameter by EM [nm] & $31-74$ \\
Hydrodynamic VLP diameter by DLS [nm] & $73(\text { PDl: } 0.04)^{(b)}$ \\
Buoyant density $\left[g \mathrm{~cm}^{-3}\right]$ & $1.14-1.15$
\end{tabular}

${ }^{(a)}$ Estimated based on determination of $O D_{600}$.

${ }^{(b)}$ Product-containing fraction after analysis by HP-SEC.

detect dS expression in the fusion protein. Co-localization of CSP and dS signals was observed in nano-scaled particles, further supporting the formation of VLP structures. We also observed some staining by the anti-CSP antibody alone (red), which was expected as the CSP portion of the fusion protein potentially masked the dS antigen recognized by the anti-dS antibody. We also used polyclonal anti-CSP antibodies to confirm the 
expression of CSP on the surface of CSP-dS VLPs by standard ELISA (Figure 3D).

\section{CSP-dS VLP Was Immunogenic and Induced Antibodies to Multiple Regions of CSP}

Mice were initially immunized with three $10 \mu \mathrm{g}$ doses of CSP-dS $\operatorname{VLP}(n=5)$ or $\operatorname{rCSP}(n=5)$ as a non-VLP comparison control, each formulated with Alhydrogel adjuvant. Sera were collected after the final dose and evaluated for antibodies by standard ELISA. Serum samples were tested between $1 / 100$ and $1 / 64000$ dilution for total IgG to full-length CSP (Figure 4A). There was a strong induction of anti-CSP IgG in all mice, apart from mouse \#51 of the rCSP vaccine group (Figure S3A). We next measured the IgG response to antigens representing the central-repeat (NANP) and C-terminal (CT) regions of CSP (Figures 4B, C). All mice demonstrated high levels of IgG to the NANP and CT antigens, apart from mouse \#51, and mouse \#48 of the rCSP vaccine group also had low reactivity to the CT (Figures S3B, C). Total IgG responses to full-length CSP, NANP and CT were comparable between groups $(\mathrm{p}=0.645, \mathrm{p}=0.508$ and $\mathrm{p}=0.289$, respectively). Although, it is noteworthy that CT-IgG was trending towards being significantly higher in the CSP-dS vaccine group at $1 / 100$ dilution $(\mathrm{p}=0.076)$ and was significantly higher when sera were tested after only two vaccine doses ( $p=0.009$; Figure S4). Antibodies to full-length CSP were further characterized for IgG subclasses (Figure 5A). Immunization with the CSP-dS VLP or rCSP predominantly induced anti-CSP IgG1, whereas IgG2a, IgG2b and IgG3 responses were variable, which is typical for vaccinations using Alhydrogel as the adjuvant. Overall, there were no significant differences in IgG subclass response between the vaccine groups ( $\mathrm{p}>0.05$ for all tests).

\section{Vaccine-Induced Antibodies Mediated Fc-Dependent Responses}

We used a range of in vitro assays to evaluate the potential protective activity of vaccine-induced antibodies. Assays were selected on the basis that the given antibody property has been associated with RTS,S vaccine efficacy. Note that we did not conduct animal challenge studies since the RTS,S CSP vaccine construct has already demonstrated protective efficacy in multiple clinical trials. We examined whether vaccine-induced antibodies could mediate Fc-dependent responses, which have been correlated with RTS,S vaccine efficacy (12), using established plate-based detection assays (Figure 5B). Firstly, we measured the ability of anti-CSP antibodies to fix human complement protein, C1q. Clq-fixation is essential to activate the classical complement pathway, and has been previously identified as a mechanism of naturally-acquired and vaccineinduced immunity to $P$. falciparum sporozoites $(30,40)$. The ability of vaccine-induced antibodies to fix $\mathrm{Clq}$ was moderate and did not significantly differ between vaccine groups $(\mathrm{p}=0.530$, Figure 5B). We then measured whether the antibodies could effectively form immune complexes to CSP that interact with dimeric recombinant human Fc $\gamma$ RIIa, as a functional surrogate

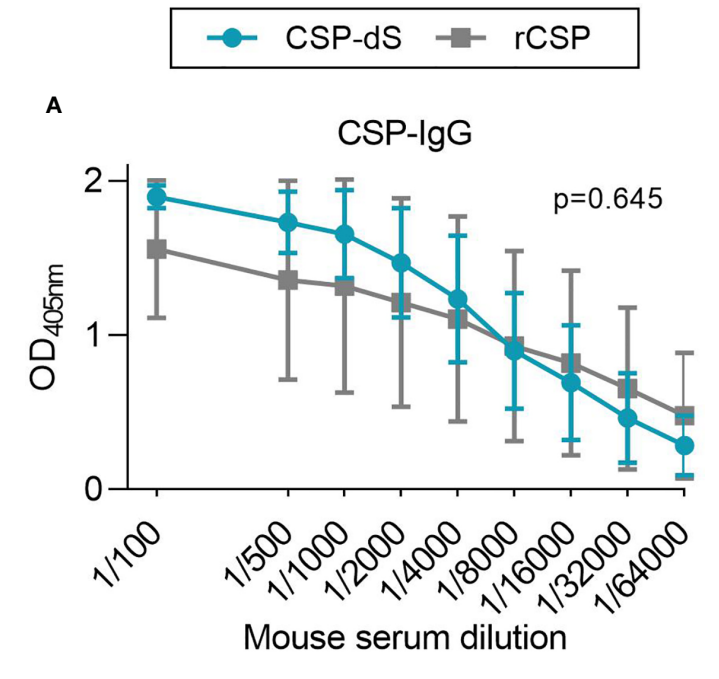

BANP-lgG
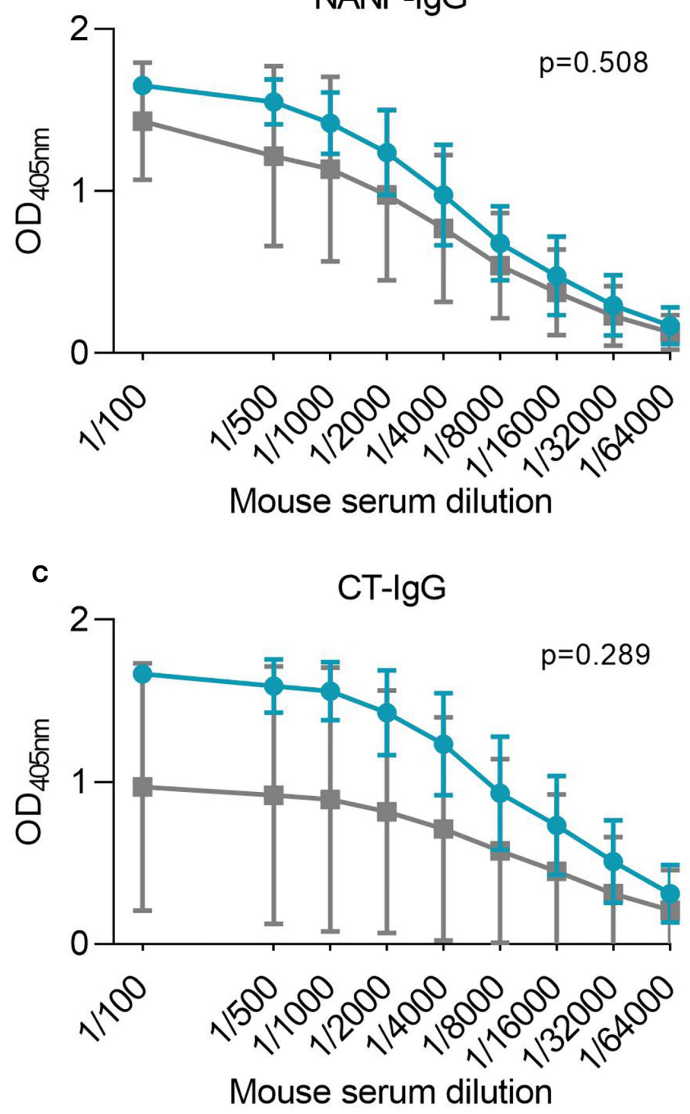

FIGURE 4 | CSP-dS is immunogenic in mice. Swiss mice were immunized with three $10 \mu \mathrm{g}$ doses of CSP-dS ( $n=5$; circles) or rCSP as a control $(n=5$; squares). Serum samples collected after the final immunization were tested for total lgG to (A) full-length CSP, and antigens representing the (B) centralrepeat (NANP) and $\mathbf{( C )} \mathrm{C}$-terminal $(\mathrm{CT})$ regions of CSP. The $\mathrm{x}$-axis is presented on a log2 scale and the mean and standard deviation of two independent experiments are shown. The AUC for each mouse in CSP-dS and rCSP vaccine groups were compared using the unpaired t-test. 


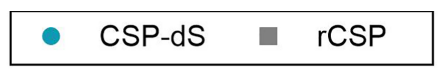

A
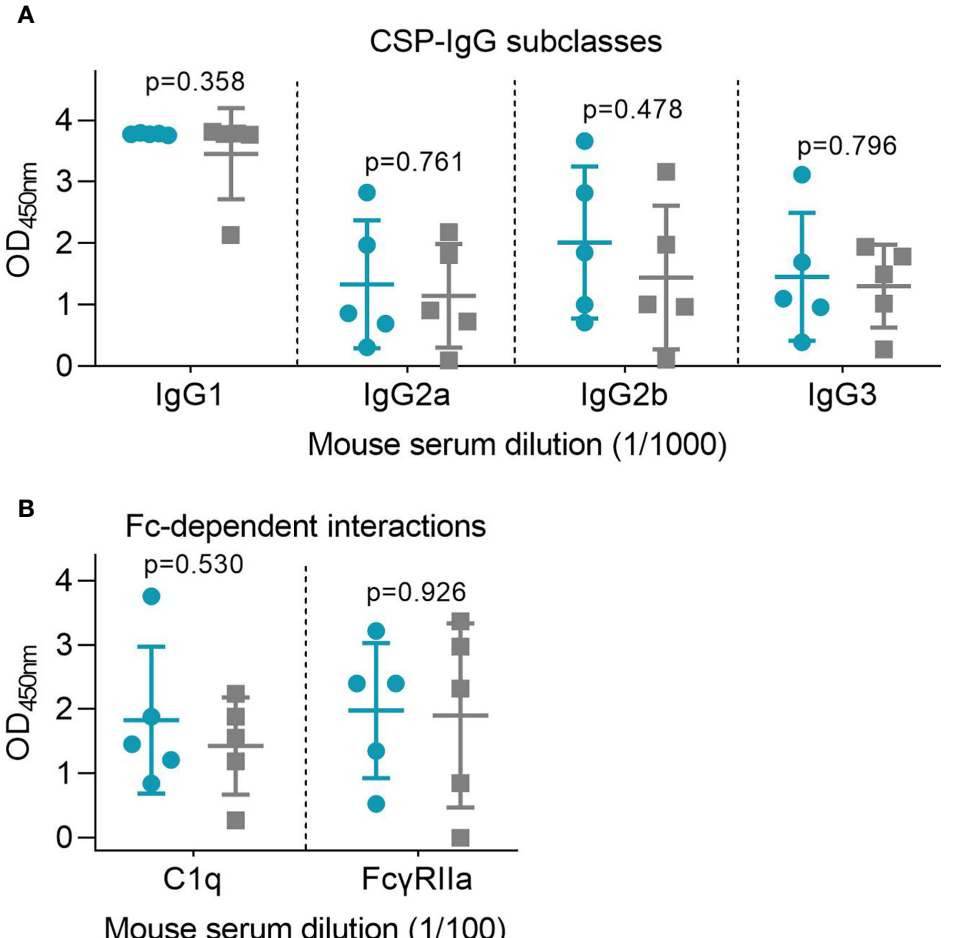

FIGURE 5 | Immunization with CSP-dS induces different IgG subclasses and Fc-dependent antibody responses. Swiss mice were immunized with three 10 $\mu \mathrm{g}$ doses of CSP-dS ( $n=5$; circles) or rCSP as a control ( $n=5$; squares). Serum samples collected after the final immunization were tested for (A) anti-CSP IgG subclass responses and (B) FC-dependent responses against full-length CSP, including C1q-fixation and binding to dimeric FcyRIla (data from one experiment). Mean and standard deviation are shown and reactivity between the CSP-dS and rCSP vaccine groups were compared using the unpaired t-test.

for antibody cross-linking of FcyR expressed on immune cells $(12,32,33)$. Fc $\gamma$ RIIa is widely expressed on different immune cells, such as monocytes and neutrophils, and can interact with antibodies to promote opsonic phagocytosis. Importantly, Fc $\gamma \mathrm{R}$ binding and opsonic phagocytosis are known functional mechanisms of antibodies to the CSP antigen (12). There was a moderate level of FcyRIIa-binding that was comparable between vaccine groups $(p=0.926$, Figure 5B). However, it should be noted that mouse IgG subclasses do not have same potential to fix human complement and bind human FcyRs as seen with human IgG subclasses induced by malaria vaccines, which are typically predominantly IgG1 and IgG3 (41). Furthermore, the predominant IgG subclass induced in mice was IgG1, which has limited activity to fix murine complement or interact with FcyRs.

We also evaluated the correlations between all antibody variables obtained from the CSP-dS and rCSP vaccine groups, as shown in Table 2. Total IgG responses to full-length CSP and the NANP and CT antigens strongly and significantly correlated with each other $(\mathrm{p}<0.05$ for all tests). C1q-fixation was

TABLE 2 | Correlations between antibody responses in mice after three $10 \mu \mathrm{g} / \mathrm{dose}$ immunizations with CSP-dS or rCSP vaccines ( $\mathrm{n}=10$ ).

\begin{tabular}{|c|c|c|c|c|c|c|c|c|}
\hline & NANP & CT & IgG1 & IgG2a & IgG2b & IgG3 & C1q & FcyR \\
\hline CSP & 0.977 & 0.791 & 0.917 & 0.519 & 0.663 & 0.644 & 0.640 & 0.781 \\
\hline NANP & & 0.703 & 0.955 & 0.458 & 0.615 & 0.581 & 0.598 & 0.735 \\
\hline CT & & & 0.664 & 0.567 & 0.647 & 0.581 & 0.607 & 0.641 \\
\hline IgG1 & & & & 0.464 & 0.501 & 0.486 & 0.524 & 0.586 \\
\hline $\operatorname{lgG} 2 a$ & & & & & 0.627 & 0.906 & 0.934 & 0.214 \\
\hline $\lg G 2 b$ & & & & & & 0.656 & 0.780 & 0.553 \\
\hline IgG3 & & & & & & & 0.953 & 0.423 \\
\hline C1q & & & & & & & & 0.380 \\
\hline
\end{tabular}

Total IgG responses to CSP, NANP and CT antigens; IgG subclass, C1q and FCYR responses to CSP. Pearson's correlation coefficients are shown, and significant values ( $\mathrm{p}<0.05)$ are highlighted in grey. 
significantly correlated with IgG to CSP and not the NANP or CT antigens, specifically with the IgG2a, IgG2b and IgG3 subclasses. In contrast, FcyRIIa-binding correlated with total IgG to all three antigens tested (CSP, NANP and CT), but did not correlate with any specific IgG subclass. C1q-fixation and Fc $\gamma$ RIIa-binding responses were not significantly correlated $(\mathrm{p}=0.278)$, although it should be noted that the sample size was relatively small $(n=10)$, so the power to detect correlations of weak to moderate strength was limited.

\section{CSP-dS Immunization Induced Antibodies With Protective Characteristics}

To further assess the immune responses generated, we benchmarked CSP-dS induced antibody responses with characteristics known to be associated with protection against malaria. Studies have shown that $\sim 200 \mu \mathrm{g} / \mathrm{ml}$ of NANP-specific antibodies confers protection against malaria infection in mouse models (42), and $114 \mu \mathrm{g} / \mathrm{ml}$ of antibodies are induced by RTS,S vaccination in protected participants (43). To establish whether CSP-dS induced antibodies above the reported protective thresholds, we estimated the serum concentration of NANP-IgG using the mouse $2 \mathrm{~A} 10 \mathrm{mAb}$ to generate a standard curve (Figure S5). 2A10 targets the central-repeat NANP region of CSP and confers protection against experimental malaria infection in mice (42). Immunization with the CSP-dS induced high antibody concentrations that exceeded the reported protective thresholds (mean, $942 \mu \mathrm{g} / \mathrm{ml}$; standard deviation (SD), $847 \mu \mathrm{g} / \mathrm{ml}$ ) and were greater than that measured in the rCSP vaccine group (mean, 638 $\mu \mathrm{g} / \mathrm{ml}$; SD, $565 \mu \mathrm{g} / \mathrm{ml}$, Figure 6A). Furthermore, a pooled sample of the CSP-dS vaccine group demonstrated strong IgG avidity, similar to that of the protective $2 \mathrm{~A} 10 \mathrm{mAb}$ (Figure 6B).

To confirm the CSP-dS vaccine-induced antibodies recognized a protective epitope of CSP, or bound to CSP with specificity and avidity similar to known protective antibodies, we performed a competition ELISA using the human MGG4 mAb, which confers protection in mouse passive immunization studies (36). Samples from individual mice substantially inhibited the binding of MGG4 to the NANP peptide (mean, $48 \%$; SD, $7 \%$, Figure 6C).

\section{Fractional Dosing of CSP-dS Was Immunogenic in Mice}

To determine whether the CSP-dS VLP was immunogenic at a lower dose, we performed a second immunization study. Mice were immunized with three $2 \mu \mathrm{g}$ doses of CSP-dS, which was a fraction $\left(1 / 5^{\text {th }}\right)$ of the original dosage administered in the first vaccine study. Serum samples collected after the final immunization were evaluated by standard ELISA, and all mice demonstrated strong IgG responses to full-length CSP, NANP and CT antigens (Figures 7A-C). Therefore, CSP-dS was highly immunogenic, even when administered at a lower dose.

\section{DISCUSSION}

New vaccine platforms could facilitate the development and licensure of highly efficacious malaria vaccines. Here, we describe a novel technique to produce VLPs using the small surface antigen (dS) of duck hepatitis B virus as a scaffold to express malaria antigens for vaccine development. As a proof-ofconcept, we used the RTS,S vaccine construct of CSP as the model antigen since RTS,S has shown reproducible efficacy in human clinical trials, which is mediated by vaccine-induced antibodies. Further, CSP-based vaccines have shown reproducible protective efficacy in animal models (5). Our CSP-dS fusion protein resulted in particle formation without the need for excess unfused $\mathrm{dS}$, and thus a higher density of CSP was displayed on the VLP surface compared to conventional VLP-based vaccine approaches. The CSP-dS VLP was immunogenic in mice and induced antibody responses to multiple regions of the CSP, even when administered at lower doses. To understand the protective potential of vaccine-induced antibodies, we performed specific in vitro assays. We found that vaccine-induced antibodies demonstrated substantial Fcdependent functional activities, had avidity comparable to a protective murine $\mathrm{mAb}$, and could out-compete the binding of a human $\mathrm{mAb}$ that protects against malaria in a mouse challenge model $(36,42)$.
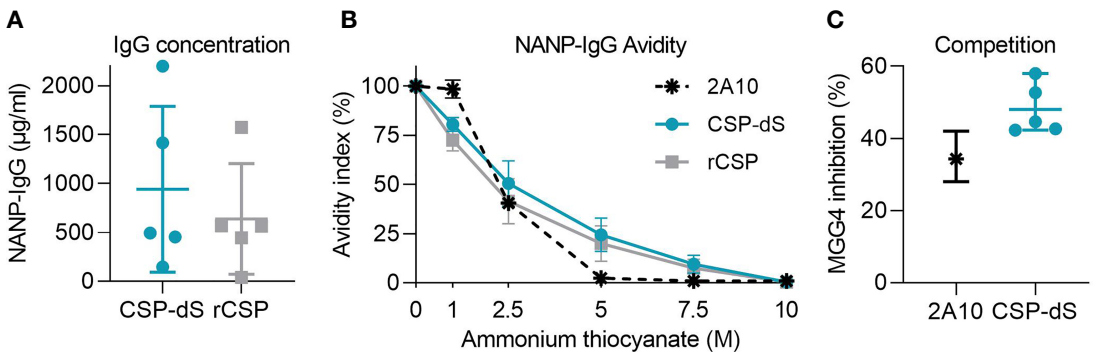

FIGURE 6 | Protective characteristics of CSP-dS induced antibodies. Individual/pooled samples were collected from mice immunized with three $10 \mu \mathrm{g}$ doses of CSP-dS or rCSP. (A) Serum concentration of NANP-IgG in CSP-dS and rCSP vaccine groups. Mean and SD of one experiment testing the individual samples at three dilutions to calculate antibody concentration. (B) NANP-lgG avidity index in pooled samples was calculated in the presence of 0-10 $\mu \mathrm{g} / \mathrm{ml}$ ammonium thiocyanate. (C) Antibody samples (CSP-dS and 2A10 mAb) moderately inhibited the binding of protective human mAb, MGG4, in a competition NANP-IgG ELISA. Mean and range of two independent experiments are shown. 


\section{$53 \multimap 54 \rightarrow 55 \multimap 56 \rightarrow 57$}

A

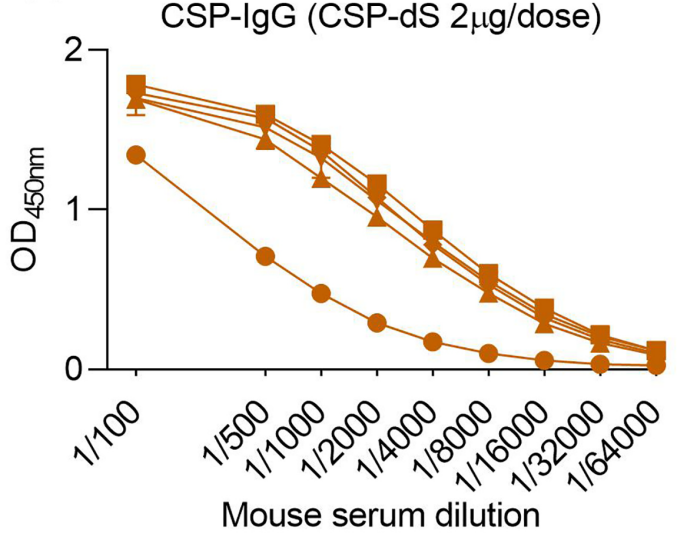

B

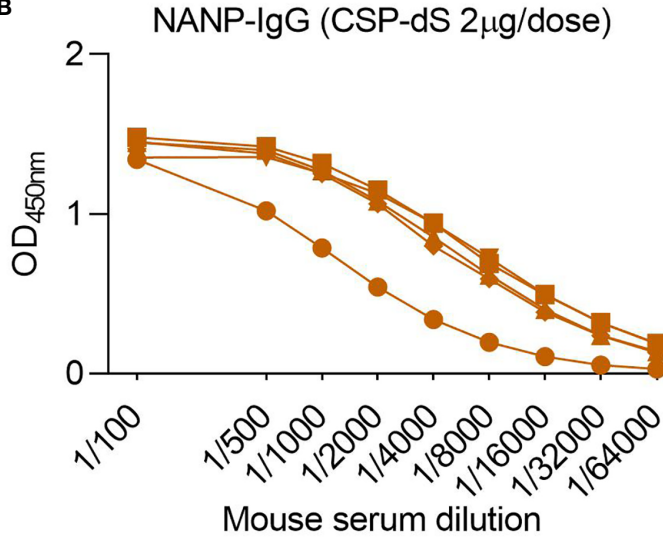

C

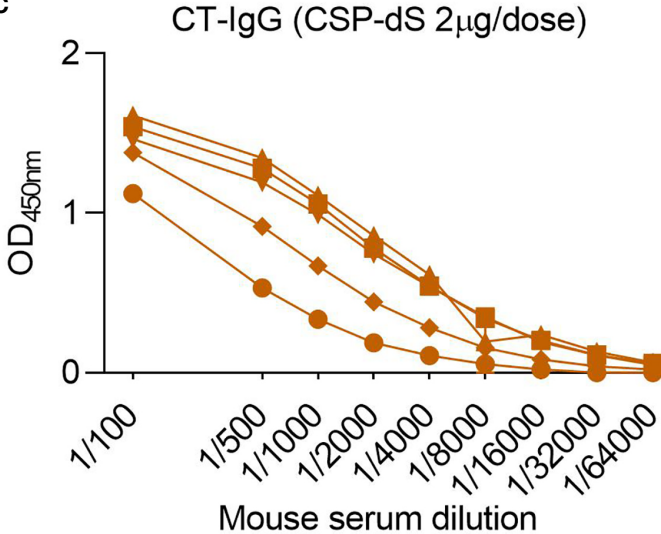

FIGURE 7 | Fractional dosing of CSP-dS is immunogenic in mice. C57/BL6 mice were immunized with three $2 \mu \mathrm{g}$ doses of CSP-dS (mouse \#53-57). Serum samples collected after the final immunization were tested for total lgG to (A) full-length CSP, and antigens representing the (B) central-repeat (NANP) and (C) C-terminal (CT) regions of CSP. The x-axis is presented on a log2 scale and the mean and range of two independent experiments are shown.
We report for the first time, the production of a dS-based VLP that formed particles in the absence of excess unfused scaffold protein $(44,45)$. Previously, we reported the expression of malaria transmission-blocking vaccine antigens $\operatorname{Pfs} 230$ and Pfs 25 as VLPs using the duck HBV platform $(19,21)$. However, in those cases, the VLPs were formed with excess dS protein and, as a result, only a minority of the total protein content was formed by the vaccine antigen. The CSP-dS fusion protein successfully formed particulate structures ranging from 31-74 $\mathrm{nm}$ as visualized by TEM. We also confirmed the display of CSP on the CSP-dS VLP, and importantly did not detect any unfused $\mathrm{dS}$ or degradation productions by Western blot. While the absence of unfused dS made preparation of the CSP-dS VLP a special case, the resulting dimensions and buoyant density were similar to previous dS-based VLP developments $(19,20)$. Transfer of the analytical purification process to a scalable downstream process for vaccine production represents an important task for the future and may improve the VLP product yield per biomass $\left(\mathrm{Y}_{\mathrm{P} / \mathrm{X}}\right)$. Nevertheless, considering the CSP-dS VLP was exclusively formed by the fusion protein, the yield per biomass of $\sim 0.2 \mathrm{mg} \mathrm{g}^{-1}$ was remarkable. We additionally generated a prototrophic RB11-based strain, which performed like the Der\#949 strain regarding CSP-dS production (data not shown) but would be more suited for future scale-up due to simpler nutrient requirements. The CSP-dS VLP vaccine is likely to be suited to formulation with a range of different adjuvants, or to be co-formulated with other vaccine components, such as other malaria antigens, in multivalent vaccines. However, further studies will be needed to assess specific antigen combinations and formulations.

The CSP-dS VLP was immunogenic in mice and induced antibody responses to full-length CSP and antigens representing the central-repeat and $\mathrm{C}$-terminal regions of the protein. Notably, the CSP-dS construct was based on a truncated form of CSP including the central-repeat and C-terminal regions, similar to the RTS,S vaccine construct. However, antibodies to the N-terminal region of CSP can inhibit sporozoite function in vitro, and the passive transfer of $\mathrm{N}$-terminal antibodies can confer protection against malaria in murine model (46-49). Therefore, inclusion of the N-terminal region may be favorable for future CSP-based vaccines, or selected epitopes such as the junctional epitope located between the N-terminal and centralrepeat regions $(36,49)$. Additional modifications could also be made in the central-repeat region, which is comprised of $\sim 37$ NANP tandem repeats. Indeed, vaccine constructs with fewer repeats can modulate the immune response to other regions of CSP when given to mice (50). Furthermore, it was recently shown that mice vaccinated with a VLP encoding only 9 NANP repeats could induce potent anti-CSP antibodies that fixed complement (51).

CSP-dS vaccine-induced antibodies demonstrated Fcdependent functional activities including complement-fixation and FcrRIIa-binding. While human IgG1 is cytophilic and potently activates complement and promotes opsonic 
phagocytosis (and other Fc-dependent effector functions), murine IgG1 is strikingly different and has little functional activity (41). The complement fixing and Fc $\gamma \mathrm{R}$-binding activity in this study was likely due to IgG2b and IgG3, which were induced at lower levels. Our study evaluated antibody Fcdependent interactions with complement and Fc $\gamma R$, but additional functions such as antibody-mediated invasion inhibition should also be explored in future studies. However, currently the function of hepatocyte invasion inhibition has not been shown to correlate with protection with the RTS,S vaccine (5). It is likely that vaccine-induced antibodies measured in our study are active in invasion inhibition since it is known that antiNANP IgG can inhibit sporozoite invasion, the antibodies had similar avidity to $2 \mathrm{~A} 10$, which can inhibit invasion (52), and antibodies out-competed MAb MGG4, which has invasioninhibitory activity (36).

We used Alhydrogel as an adjuvant because it has been extensively used in human vaccination, but alternative adjuvants should be further investigated that may alter the IgG subclass response and possibly enhance antibody functional activity. Studies on the recently described R21 vaccine have compared the use of different adjuvants in mouse immunization studies (17). R21 is another VLP-based vaccine similar to RTS,S, but is generated using a different expression system and does not require excess unfused HBsAg scaffold protein to form particles. R21 administered with Alhydrogel induced the lowest antibody titers in BALB/c mice, while oil-in-water emulsion and saponin-based adjuvants induced higher antibody titers that were comparable (17). Interestingly, only the latter formulation conferred protection against sporozoite challenge. This suggested that differences in protective efficacy were not explained by differences in IgG titer but may have been due to differences in IgG subclass or antibody function, which were not explored.

Mice immunized with CSP-dS VLP or rCSP as a non-VLP control had comparable antibody responses to full-length CSP and the central-repeat region. However, antibodies to the C-terminal region were significantly higher in the CSP-dS group after 2 doses, in which 5/5 mice had high levels of anti-CT IgG, while for the rCSP group only $3 / 5$ mice had strong reactivity. We anticipated to see higher antibody responses in the CSP-dS group because VLPs are generally considered more immunogenic than protein as they are larger in size, and therefore optimal for uptake by antigen presenting cells and inducing adaptive immune responses (53). Indeed, the CSP-dS VLP was large in size and formed particles $\sim 3$ times greater than related CSP-based vaccines, RTS,S and R21. Although, we must take caution when directly comparing the CSPdS VLP and rCSP vaccines because even though both were administered at $10 \mu \mathrm{g}$ doses, the amount of CSP present in the CSP-dS VLP would have only been $\sim 5.2 \mu \mathrm{g}$ (as the fusion protein was comprised of $\sim 52 \%$ CSP). Therefore, the CSP-dS VLP may have been superior to the rCSP vaccine, given that the amount of CSP administered was lower overall. We performed a second immunization study and found that even at a lower concentration of $2 \mu \mathrm{g}$ per dose, the CSP-dS VLP strongly induced antibodies to full-length CSP and the central-repeat and C-terminal regions in 5/5 mice. We did not undertake a formal comparison of the immunogenicity of CSP-dS VLPs versus rCSP since studies in humans have already reported superior immunogenicity of VLPs and other nanoparticle vaccines. Furthermore, the rCSP used as a non-VLP control vaccine was based on full-length CSP whereas the CSP-dS VLP was based on a truncated form of CSP, which also limited a direct comparison. Formal assessment of immunogenicity would be a part of any potential future development work.

Early studies found that RTS,S administered with alum was poorly efficacious against controlled human malaria infection in healthy volunteers (16). However, vaccine efficacy was enhanced when RTS,S was administered with an oil-in-water emulsion of MPL and QS-21, which has since been modified to a liposomebased adjuvant known as AS01 and is the current RTS,S vaccine adjuvant (8). This raises an important question of what the true advantages are of using a VLP-based vaccine instead of recombinant protein, and whether enhanced immunogenicity and efficacy are largely due to the adjuvant itself (5). It is difficult to draw a clear conclusion, as many studies do not include a nonVLP vaccine as a negative control.

There are interesting similarities and differences between our CSP-dS VLP and the related R21 and RTS,S vaccines. Notably, CSP-dS and R21 are both comprised of a fusion protein between CSP and scaffold protein that can form particles without the need for excess unfused scaffold protein (17). This may be advantageous as CSP represents a larger portion of the final vaccine construct in comparison to the RTS,S vaccine that is largely comprised of scaffold protein (16). Another possible advantage of our CSP-dS VLP is that the dS scaffold protein is derived from duck hepatitis $B$ virus rather than human hepatitis B virus (HBV), which is used for R21 and RTS,S. The dS scaffold protein forms larger particles that may be more effective at inducing immune responses (53), and the CSP-dS VLPs were $\sim 3$ times larger than the R21 and RTS,S VLPs (17). Furthermore, the dS scaffold may also be favorable as vaccine recipients should not have pre-existing antibodies to the duck HBsAg. In some settings, it may not be desirable to use a malaria vaccine that includes HBsAg in populations who have already received $\mathrm{HBV}$ vaccines. $\mathrm{HBV}$ vaccines are recommended at birth in many regions, whereas malaria vaccines would be given later in infancy. It is unclear whether pre-existing serum antibodies to the human HBsAg would interfere with vaccine-induced responses, but this has been reported for serum antibodies to CSP $(42,54)$. For these reasons, our CSP-dS VLP may be advantageous compared to other particle-based approaches, although a direct comparison would be needed to confirm which vaccine is superior.

In summary, we have demonstrated a novel approach to develop VLP structures without the co-expression of unfused dS scaffold protein. We produced a CSP-dS fusion protein that successfully formed homogenous particles and correctly displayed CSP on the VLP surface. Furthermore, the CSP-dS VLP was immunogenic in mice and induced potent IgG responses to multiple regions of the CSP antigen, which demonstrated functional Fc-dependent functional activities, had high avidity, and could out-compete a known protective $\mathrm{mAb}$. Our platform to produce VLPs formed with the fusion 
protein only, without the need for excess scaffolding protein, is highly novel and warrants further evaluation in pre-clinical efficacy studies for CSP and other candidate malaria vaccine antigens.

\section{DATA AVAILABILITY STATEMENT}

The original contributions presented in the study are included in the article/Supplementary Material. Further inquiries can be directed to the corresponding authors.

\section{ETHICS STATEMENT}

The animal study was reviewed and approved by Animal Ethics Committee of the Walter and Eliza Hall Institute.

\section{AUTHOR CONTRIBUTIONS}

LK performed immunoassays for both mouse immunization studies and data analysis. DW generated, purified, and characterized the CSP-dS VLP. LR performed immunoassays for the first mouse immunization study. DD generated recombinant proteins used in immunoassays. $\mathrm{CP}, \mathrm{BK}, \mathrm{EH}$ and $\mathrm{J}-\mathrm{AC}$ visualized the VLPs using N-SIM and TEM. BW and PH generated reagents for immunoassays. J-AC and DD performed SDS-PAGE and Western blotting analyses. LK, DW, MS, VJ, MP, $\mathrm{J}$-AC and JB were involved in the study design. LK, DW, J-AC and JB wrote the manuscript, which was reviewed and approved by all authors. All authors contributed to the article and approved the submitted version.

\section{REFERENCES}

1. World Health Organization. World malaria report 2019. (2019). Available at: https://www.who.int/publications/i/item/9789241565721.

2. Ashley EA, Dhorda M, Fairhurst RM, Amaratunga C, Lim P, Suon S, et al. Spread of artemisinin resistance in Plasmodium falciparum malaria. $N$ Engl $J$ Med (2014) 371(5):411-23. doi: 10.1056/NEJMoa1314981

3. Rogerson SJ, Beeson JG, Laman M, Poespoprodjo JR, William T, Simpson JA, et al. Identifying and combating the impacts of COVID-19 on malaria. BMC Med (2020) 18(1):1-7. doi: 10.1186/s12916-020-01710-x

4. World health Organization. Global technical strategy for malaria 2016-2030. Massachusetts Medical Society (2015).

5. Beeson JG, Kurtovic L, Dobaño C, Opi DH, Chan J-A, Feng G, et al. Challenges and strategies for developing efficacious and long-lasting malaria vaccines. Sci Transl Med (2019) 11(474):eaau1458. doi: 10.1126/ scitranslmed.aau1458

6. Casares S, Brumeanu T-D, Richie TL, The RTS. S malaria vaccine. Vaccine (2010) 28(31):4880-94. doi: 10.1016/j.vaccine.2010.05.033

7. Kester KE, Cummings JF, Ofori-Anyinam O, Ockenhouse CF, Krzych U, Moris $\mathrm{P}$, et al. Randomized, double-blind, phase 2a trial of falciparum malaria vaccines RTS, S/AS01B and RTS, S/AS02A in malaria-naive adults: safety, efficacy, and immunologic associates of protection. J Infect Dis (2009) 200 (3):337-46. doi: 10.1086/600120

8. RTSS Clinical Trial Partnerships. Efficacy and safety of RTS,S/AS01 malaria vaccine with or without a booster dose in infants and children in Africa: final results of a phase 3, individually randomised, controlled

\section{FUNDING}

This work was funded by the National Health and Medical Research Council (NHMRC) of Australia (Senior Research Fellowship, Program Grant, and Investigator Grant to JB), the Australian Government Research Training Program Scholarship to LK, the Jim and Margaret Beever Fellowship (Burnet Institute) to J-AC and project grant (GNT1145303) to PH and BW. Burnet Institute is supported by funding from the NHMRC Independent Research Institutes Infrastructure Support Scheme and a Victorian State Government Operational Infrastructure grant. LK, LR, J-AC, and JB are supported by the NHMRCfunded Australian Centre for Research Excellence on Malaria Elimination.

\section{ACKNOWLEDGMENTS}

The authors gratefully acknowledge Brigitte Derksen for technical assistance and Jack Richards for providing mouse IgG subclass reagents. Recombinant full length CSP was kindly provided by PATH Malaria Vaccine Initiative (USA) and Gennova Biopharmaceuticals (India). We also acknowledge Monash University and Rob J Center for producing the 2A10 and MGG4 mAbs (respectively), and Gaoqian Feng for validating the mAbs.

\section{SUPPLEMENTARY MATERIAL}

The Supplementary Material for this article can be found online at: https://www.frontiersin.org/articles/10.3389/fimmu.2021. 641421/full\#supplementary-material

trial. Lancet (2015) 386(9988):31-45. doi: 10.1016/S0140-6736(15) 60721-8

9. RTSS Clinical trials Partnership. Efficacy and safety of the RTS, S/AS01 malaria vaccine during 18 months after vaccination: a phase 3 randomized, controlled trial in children and young infants at 11 African sites. PLoS Med (2014) 11:e1001685. doi: 10.1371/journal.pmed.1001685

10. Adepoju P. RTS, S malaria vaccine pilots in three African countries. Lancet (2019) 393(10182):1685. doi: 10.1016/S0140-6736(19)30937-7

11. White MT, Verity R, Griffin JT, Asante KP, Owusu-Agyei S, Greenwood B, et al. Immunogenicity of the RTS, S/AS01 malaria vaccine and implications for duration of vaccine efficacy: secondary analysis of data from a phase 3 randomised controlled trial. Lancet Infect Dis (2015) 15(12):1450-8. doi: 10.1016/S1473-3099(15)00239-X

12. Kurtovic L, Atre T, Feng G, Wines BD, Chan JA, Boyle MJ, et al. Multi-functional antibodies are induced by the RTS,S malaria vaccine and associated with protection in a phase I/IIa trial. J Infect Dis (2020), jiaal44. doi: 10.1101/851725

13. Suscovich TJ, Fallon JK, Das J, Demas AR, Crain J, Linde CH, et al. Mapping functional humoral correlates of protection against malaria challenge following RTS, S/AS01 vaccination. Sci Transl Med (2020) 12(553): eabb4757. doi: 10.1126/scitranslmed.abb4757

14. Thompson HA, Hogan AB, Walker PG, White MT, Cunnington AJ, Ockenhouse $\mathrm{CF}$, et al. Modelling the roles of antibody titre and avidity in protection from Plasmodium falciparum malaria infection following RTS, S/AS01 vaccination. Vaccine (2020) 38(47):7498-507. doi: 10.1016/j.vaccine.2020.09.069

15. Dobaño C, Sanz H, Sorgho H, Dosoo D, Mpina M, Ubillos I, et al. Concentration and avidity of antibodies to different circumsporozoite 
epitopes correlate with RTS, S/AS01E malaria vaccine efficacy. Nat Commun (2019) 10(1):2174. doi: 10.1038/s41467-019-10195-z

16. Gordon D, McGovern T, Krzych U, Cohen J, Schneider I, LaChance R, et al. Safety, immunogenicity, and efficacy of a recombinantly produced Plasmodium falciparum circumsporozoite protein-hepatitis B surface antigen subunit vaccine. J Infect Dis (1995) 171(6):1576-85. doi: 10.1093/infdis/171.6.1576

17. Collins KA, Snaith R, Cottingham MG, Gilbert SC, Hill AV. Enhancing protective immunity to malaria with a highly immunogenic virus-like particle vaccine. Sci Rep (2017) 7:46621. doi: 10.1038/srep46621

18. Bachmann MF, Jennings GT. Vaccine delivery: a matter of size, geometry, kinetics and molecular patterns. Nat Rev Immunol (2010) 10(11):787. doi: 10.1038/nri2868

19. Wetzel D, Chan J, Suckow M, Barbian A, Weniger M, Jenzelewski V. Display of malaria transmission-blocking antigens on chimeric duck hepatitis $B$ virusderived virus-like particles produced in Hansenula polymorpha. PLoS One (2019) 14(9):e0221394. doi: 10.1371/journal.pone.0221394

20. Wetzel D, Rolf T, Suckow M, Kranz A, Barbian A, Chan J-A, et al. Establishment of a yeast-based VLP platform for antigen presentation. Microbial Cell Fact (2018) 17(1):17. doi: 10.1186/s12934-018-0868-0

21. Chan J-A, Wetzel D, Reiling L, Miura K, Drew DR, Gilson PR, et al. Malaria vaccine candidates displayed on novel virus-like particles are immunogenic and induce transmission-blocking activity. PloS One (2019) 14(9):e0221733. doi: 10.1371/journal.pone.0221733

22. De Wilde M, Cohen J. Hybrid protein between CS from plasmodium and HBsAg. Google Patents (1999).

23. Degelmann A, Müller F, Sieber H, Jenzelewski V, Suckow M, Strasser AW, et al. Strain and process development for the production of human cytokines in Hansenula polymorpha. FEMS Yeast Res (2002) 2(3):349-61. doi: 10.1016/ S1567-1356(02)00096-X

24. Lahtchev KL, Semenova VD, Tolstorukov II, van der Klei I, Veenhuis M. Isolation and properties of genetically defined strains of the methylotrophic yeast Hansenula polymorpha CBS4732. Arch Microbiol (2002) 177(2):150-8. doi: 10.1007/s00203-001-0370-6

25. Faber KN, Haima P, Harder W, Veenhuis M, Geert A. Highly-efficient electrotransformation of the yeast Hansenula polymorpha. Curr Genet (1994) 25(4):305-10. doi: 10.1007/BF00351482

26. Guengerich L, Kang H, Behle B, Gellissen G, Suckow M. A platform for heterologous gene expression based on the methylotrophic yeast Hansenula polymorpha. Genet Biotechnol: Springer (2004) 2:273-87. doi: 10.1007/978-3662-07426-8_14

27. Kastenmüller K, Espinosa DA, Trager L, Stoyanov C, Salazar AM, Pokalwar S, et al. Full-length Plasmodium falciparum circumsporozoite protein administered with long-chain poly (I. C) or the Toll-like receptor 4 agonist glucopyranosyl lipid adjuvant-stable emulsion elicits potent antibody and CD4+ T cell immunity and protection in mice. Infect Immun (2013) 81 (3):789-800. doi: 10.1128/IAI.01108-12

28. Schaefer S, Piontek M, Ahn S, Papendieck A, Janowicz Z, Timmermans I, et al. Recombinant hepatitis $B$ vaccines-disease characterization and vaccine production. Hansenula Polymorpha-biol Appl (2002) 1:175-210. doi: 10.1002/3527602356.ch12

29. Kurtovic L, Agius PA, Feng G, Drew DR, Ubillos I, Sacarlal J, et al. Induction and decay of functional complement-fixing antibodies by the RTS, S malaria vaccine in children, and a negative impact of malaria exposure. BMC Med (2019) 17(1):45. doi: 10.1186/s12916-019-1277-x

30. Kurtovic L, Behet MC, Feng G, Reiling L, Chelimo K, Dent AE, et al. Human antibodies activate complement against Plasmodium falciparum sporozoites, and are associated with protection against malaria in children. BMC Med (2018) 16(1):61. doi: 10.1186/s12916-018-1054-2

31. Reiling L, Boyle MJ, White MT, Wilson DW, Feng G, Weaver R, et al. Targets of complement-fixing antibodies in protective immunity against malaria in children. Nat Commun (2019) 10(1):610. doi: 10.1038/s41467019-08528-z

32. Feng G, Wines B, Kurtovic L, Chan J, Boeuf P, Mollard V, et al. Mechanisms and targets of Fc $\gamma$-receptor mediated immunity to malaria sporozoites. Nat Commun (2021).

33. Wines BD, Vanderven HA, Esparon SE, Kristensen AB, Kent SJ, Hogarth PM. Dimeric FcyR ectodomains as probes of the Fc receptor function of anti- influenza virus IgG. J Immunol (2016) 197(4):1507-16. doi: 10.4049/ jimmunol.1502551

34. Zavala F, Cochrane AH, Nardin EH, Nussenzweig RS, Nussenzweig V. Circumsporozoite proteins of malaria parasites contain a single immunodominant region with two or more identical epitopes. J Exp Med (1983) 157(6):1947-57. doi: 10.1084/jem.157.6.1947

35. Boyle M, Chan J, Handayuni I, Reiling L, Feng G, Hilton A, et al. IgM in human immunity to Plasmodium falciparum malaria. Sci Adv (2019) 5(9): eaax4489. doi: 10.1126/sciadv.aax4489

36. Tan J, Sack BK, Oyen D, Zenklusen I, Piccoli L, Barbieri S, et al. A public antibody lineage that potently inhibits malaria infection through dual binding to the circumsporozoite protein. Nat Med (2018) 24(4):401-7. doi: 10.1038/ $\mathrm{nm} .4513$

37. Mugyenyi CK, Elliott SR, McCallum FJ, Anders RF, Marsh K, Beeson JG. Antibodies to polymorphic invasion-inhibitory and non-inhibitory epitopes of Plasmodium falciparum apical membrane antigen 1 in human malaria. PLoS One (2013) 8(7):e68304. doi: 10.1371/journal.pone.0068304

38. Cova L, Abdul F, Buronfosse T. Avihepadnavirus. In: . The Springer index of viruses. New York City: Springer (2011). p. 615-24.

39. Mason W, Gerlich W, Taylor J, Kann M, Mizokami T, Loeb D, et al. Hepadnaviridae. In: Virus taxonomy. Amsterdam: Elsevier (2013). p. 445-55.

40. Behet M, Kurtovic L, Gemert G-J, Haukes C, Siebelink-Stoter R, Graumans W, et al. The complement system contributes to functional antibody-mediated responses induced by immunization with Plasmodium falciparum malaria sporozoites. Infect Immun (2018) 86:00920-17. doi: 10.1128/IAI.00920-17

41. Mestas J, Hughes CC. Of mice and not men: differences between mouse and human immunology. J Immunol (2004) 172(5):2731-8. doi: 10.4049/ jimmunol.172.5.2731

42. McNamara HA, Idris AH, Sutton HJ, Vistein R, Flynn BJ, Cai Y, et al. Antibody Feedback Limits the Expansion of B Cell Responses to Malaria Vaccination but Drives Diversification of the Humoral Response. Cell Host Microbe (2020) 28:572-85.e7. doi: 10.1016/j.chom.2020.07.001

43. Kester KE, Cummings JF, Ockenhouse CF, Nielsen R, Hall BT, Gordon DM, et al. Phase $2 \mathrm{a}$ trial of 0,1 , and 3 month and 0,7 , and 28 day immunization schedules of malaria vaccine RTS, S/AS02 in malaria-naive adults at the Walter Reed Army Institute of Research. Vaccine (2008) 26(18):2191-202. doi: 10.1016/j.vaccine.2008.02.048

44. Anderson DA, Grgacic EVL. Viral vectors expressing fusion of viral large envelope protein and protein of interest. Google Patents (2004).

45. Grgacic EVL, Anderson DA, Loke P, Anders RF. Recombinant proteins and virus like particles comprising 1 and $s$ polypeptides of avian hepadnaviridae and methods, nucleic acid constructs, vectors and host cells for producing same. Google Patents (2006).

46. Coppi A, Natarajan R, Pradel G, Bennett BL, James ER, Roggero MA, et al. The malaria circumsporozoite protein has two functional domains, each with distinct roles as sporozoites journey from mosquito to mammalian host. J Exp Med (2011) 208(2):341-56. doi: 10.1084/jem.20101488

47. Rathore D, Nagarkatti R, Jani D, Chattopadhyay R, de la Vega P, Kumar S, et al. An immunologically cryptic epitope of Plasmodium falciparum circumsporozoite protein facilitates liver cell recognition and induces protective antibodies that block liver cell invasion. J Biol Chem (2005) 280 (21):20524-9. doi: 10.1074/jbc.M414254200

48. Espinosa DA, Gutierrez GM, Rojas-López M, Noe AR, Shi L, Tse S-W, et al. Proteolytic cleavage of the Plasmodium falciparum Circumsporozoite protein is a target of protective antibodies. J Infect Dis (2015) 212(7):1111-9. doi: 10.1093/infdis/jiv154

49. Kisalu NK, Idris AH, Weidle C, Flores-Garcia Y, Flynn BJ, Sack BK, et al. A human monoclonal antibody prevents malaria infection by targeting a new site of vulnerability on the parasite. Nat Med (2018) 24(4):408. doi: 10.1038/nm.4512

50. Lewis FJ, Chatterjee D, Kaczmarski J, Gao X, Cai Y, McNamara HA, et al. Avid binding by $\mathrm{B}$ cells to the Plasmodium circumsporozoite protein repeat suppresses responses to protective subdominant epitopes. bioRxiv (2020). doi: $10.1101 / 2020.01 .12 .903682$

51. Kingston NJ, Kurtovic L, Walsh R, Joe C, Lovrecz G, Locarnini S, et al. Hepatitis B virus-like particles expressing Plasmodium falciparum epitopes induce complement-fixing antibodies against the circumsporozoite protein. Vaccine (2019) 37(12):1674-84. doi: 10.1016/j.vaccine.2019.01.056 
52. Hollingdale MR, Nardin EH, Tharavanij S, Schwartz A, Nussenzweig R. Inhibition of entry of Plasmodium falciparum and P. vivax sporozoites into cultured cells; an in vitro assay of protective antibodies. J Immunol (1984) 132(2):909-13.

53. Mohsen MO, Zha L, Cabral-Miranda G, Bachmann MF. Major findings and recent advances in virus-like particle (VLP)-based vaccines. Semin Immunol (2017) 34:123-32. doi: 10.1016/j.smim.2017.08.014

54. Kurtovic L, Boyle M, Beeson J. Epitope masking may limit antibody boosting to malaria vaccines. Immunol Cell Biol (2020) 99:126-9. doi: 10.1111/ imcb. 12415

Conflict of Interest: The authors DW, MS, VJ and MP are associated with ARTES Biotechnology GmbH which owns the license for the VLP technology.
The remaining authors declare that the research was conducted in the absence of any commercial or financial relationships that could be construed as a potential conflict of interest.

Copyright (๑) 2021 Kurtovic, Wetzel, Reiling, Drew, Palmer, Kouskousis, Hanssen, Wines, Hogarth, Suckow, Jenzelewski, Piontek, Chan and Beeson. This is an openaccess article distributed under the terms of the Creative Commons Attribution License (CC BY). The use, distribution or reproduction in other forums is permitted, provided the original author(s) and the copyright owner(s) are credited and that the original publication in this journal is cited, in accordance with accepted academic practice. No use, distribution or reproduction is permitted which does not comply with these terms. 